\title{
A Technical Overview of Technegas as a Lung Ventilation Agent
}

\author{
Geoffrey M. Currie ${ }^{1,2}$ and Dale L. Bailey ${ }^{3,4}$ \\ ${ }^{1}$ Charles Sturt University, Wagga Wagga, Australia; ${ }^{2}$ Baylor College of Medicine, Houston, Texas; ${ }^{3}$ Royal North Shore Hospital, \\ Sydney, Australia; and ${ }^{4}$ University of Sydney, Sydney, Australia
}

\begin{abstract}
CE credit: For CE credit, you can access the test for this article, as well as additional JNMT CE tests, online at https://www.snmmilearningcenter.org. Complete the test online no later than December 2024. Your online test will be scored immediately. You may make 3 attempts to pass the test and must answer $80 \%$ of the questions correctly to receive $1.0 \mathrm{CEH}$ (Continuing Education Hour) credit. SNMMI members will have their CEH credit added to their VOICE transcript automatically; nonmembers will be able to print out a CE certificate upon successfully completing the test. The online test is free to SNMMI members; nonmembers must pay $\$ 15.00$ by credit card when logging onto the website to take the test.
\end{abstract}

Technegas is a carbon-based nanoparticle developed in Australia in 1984 and has been in widespread clinical use, including SPECT imaging, since 1986 . Although ${ }^{81 \mathrm{~m}} \mathrm{Kr}$ offers the ideal ventilation properties of a true gas, Technegas is considered preferred in more than 60 countries for ventilation imaging yet has limited adoption in the United States. In March 2020, a new U.S. Food and Drug Administration application was lodged for Technegas, and the impending approval warrants a detailed discussion of the technical aspects of the technology for those for whom it is new. Technegas is a simple yet versatile system for producing highquality ${ }^{99 m}$ Tc-based ventilation studies. The design affords safety to patients and staff, including consideration of radiation and biologic risks. Technegas is the gold standard for the ventilation portion of SPECT-based ventilation-perfusion studies in pulmonary embolism and several respiratory pathologies. When approved by the U.S. Food and Drug Administration, Technegas will extend advantages to workflow, safety, and study quality for departments that adopt the technology.

Key Words: lung imaging; ventilation; Technegas

J Nucl Med Technol 2021; 49:313-319

DOI: $10.2967 /$ jnmt.121.262887

V entilation and perfusion (V/Q) lung scintigraphy has been used in the evaluation of patients with suspected pulmonary embolism for more than half a century. Although the underlying principles have not changed during this period, there have been significant advances in technology. Imaging equipment has emerged with superior resolution and sensitivity, SPECT techniques have added advantages over planar imaging alone, hybrid systems have allowed SPECT and CT to produce physiologic information coregistered with morphology, and PET and PET/CT have become more widely available. Concurrently, developments in

\footnotetext{
Received Jul. 9, 2021; revision accepted Aug. 23, 2021.

For correspondence or reprints, contact: Geoffrey M. Currie (gcurrie@csu.edu. au).

Published online September 28, 2021.

COPYRIGHT (C) 2021 by the Society of Nuclear Medicine and Molecular Imaging.
}

radiotracers have seen improved microspheres for perfusion in macroaggregated albumin and replacement of ${ }^{133} \mathrm{Xe}$ ventilation with ${ }^{99 \mathrm{~m}} \mathrm{Tc}$ aerosols, ${ }^{81 \mathrm{~m}} \mathrm{Kr}$, and Technegas (Cyclomedica Pty Ltd.), although ${ }^{133} \mathrm{Xe}$ is still used at some sites. Technegas is a carbon-based nanoparticle that has a strongly bound ${ }^{99 \mathrm{~m}} \mathrm{Tc}$ atom trapped in a cagelike structure. It was developed in Australia in 1984 (1) and began seeing widespread clinical use in Australia from 1986, and more recently including SPECT (2). Although ${ }^{81 \mathrm{~m}} \mathrm{Kr}$ offers the ideal ventilation properties of a true gas, Technegas is considered preferred in more than 60 countries for ventilation imaging yet has limited adoption in the United States. Indeed, in 1991 a Newsline article in The Journal of Nuclear Medicine (3) posed the question, "When, if ever, will the device that has won near ubiquitous use in Australia gain approval for lung ventilation studies in the U.S.?" In March 2020, a new U.S. Food and Drug Administration application was lodged for Technegas, and early in 2021 both the Society of Nuclear Medicine and Molecular Imaging and its Technologist Section, concerned about the decline in ventilation studies in response to coronavirus disease 2019 risk, requested fast tracking of Food and Drug Administration approval of Technegas. Since Technegas does not produce aerosolized particles, nor is it a gas, it allows imaging of the ventilation to the lungs to optimize patient outcomes while maintaining staff safety. The impending approval of Technegas by the U.S. Food and Drug Administration warrants a detailed discussion of the technical aspects of the technology for those for whom it is new. This discussion will not examine the broader debate on the role and application of V/Q imaging, clinical applications, or the advances in imaging technology (SPECT, SPECT/CT, and PET/CT) and the associated image interpretation. It is, however, important to note that using Technegas for ventilation imaging allows the entire V/Q protocol to move to 3-dimensional SPECT imaging with the associated improved sensitivity and specificity (4-6). SPECT performance is further enhanced with the addition of a coacquired low-dose CT scan (4-6). 


\section{LUNG VENTILATION}

Several approaches to lung ventilation studies are available in nuclear medicine, with the predominant approaches being ${ }^{81 \mathrm{~m}} \mathrm{Kr}$ as an inert gas, ${ }^{99 \mathrm{~m}} \mathrm{Tc}$-diethylenetriaminepentaacetic acid aerosol, and the ultrafine ${ }^{99 \mathrm{~m}} \mathrm{Tc}$-carbon dispersion of Technegas. ${ }^{81 \mathrm{~m}} \mathrm{Kr}$ use is limited by availability, cost, the short half-life, and the less practical SPECT. ${ }^{99 \mathrm{~m}}$ Tc-diethylenetriaminepentaacetic acid has aerosolized droplets with varying sizes $(0.5-2 \mu \mathrm{m})$, with distribution dependent on the aerodynamics of gas flow. As a result, aerosol ventilation studies can be confounded by deposition in large airways, and this issue is exacerbated in patients with respiratory symptoms. The ultrafine Technegas particles $(<0.1 \mu \mathrm{m})$, by contrast, have a gaslike distribution, particlelike retention, and the attractive properties of ${ }^{99 \mathrm{~m}} \mathrm{Tc}$ to allow high-quality imaging, including SPECT. Internationally, Technegas is considered the best alternative for the ventilation portion of the V/Q scan $(7,8)$.

\section{TECHNEGAS PRODUCTION}

Technegas is hexagonally shaped graphite capsule enclosing ${ }^{99 \mathrm{~m}} \mathrm{Tc}(1)$. The fact that these graphite particles are small $(<0.1 \mu \mathrm{m})$ and hydrophobic allows penetration deep into the airways and resists attachment to the airways, making them ideal for evaluation of deposition in the lungs (9). Technegas is distributed in the lung airways by diffusion and becomes fixed in the lumen of the airways sufficiently long to allow SPECT (10).

The commercially available Technegas generator operates with ${ }^{99 \mathrm{~m}} \mathrm{Tc}$-sodium pertechnetate eluted from the ${ }^{99} \mathrm{Mo} /{ }^{99 \mathrm{~m}} \mathrm{Tc}$ generator. The graphite crucible accommodates $0.17 \mathrm{~mL}$, and this volume should not be exceeded. Once the ${ }^{99 \mathrm{~m}} \mathrm{Tc}$ has been added to the crucible, the liquid is evaporated (simmered) at $70^{\circ} \mathrm{C}$ for $6 \mathrm{~min}$ in an ultrapure argon environment. On completion of the simmer cycle, the combustion cycle (burn) is initiated, in which an alternatingcurrent arc is produced between the terminals holding the crucible to ablate the graphite and ${ }^{99 \mathrm{~m}} \mathrm{Tc}$ (11). Temperatures of $2,750^{\circ} \mathrm{C}$ are created for $15 \mathrm{~s}$ in an ultrapure argon environment to produce the carbon nanoparticles (11). Operation of the Technegas generator is simple when the stepwise process is followed. Specific steps in the process are detailed in the user guide and will not be repeated here, but outlined below are general comments associated with each step for consideration when using the Technegas generator (Fig. 1).

\section{OPERATION OF THE TECHNEGAS GENERATOR}

The first step is to ensure that the Technegas generator is in good working order, is connected to an adequate supply of argon (or a change of argon gas bottles), is connected to the main power supply, and is powered on (Fig. 2). The delivery to the patient can occur at the patient's bedside using battery power. This option is useful because it allows the Technegas generator to be prepared in a radiation-safe location remote from patient, staff, and scanning areas (e.g.,

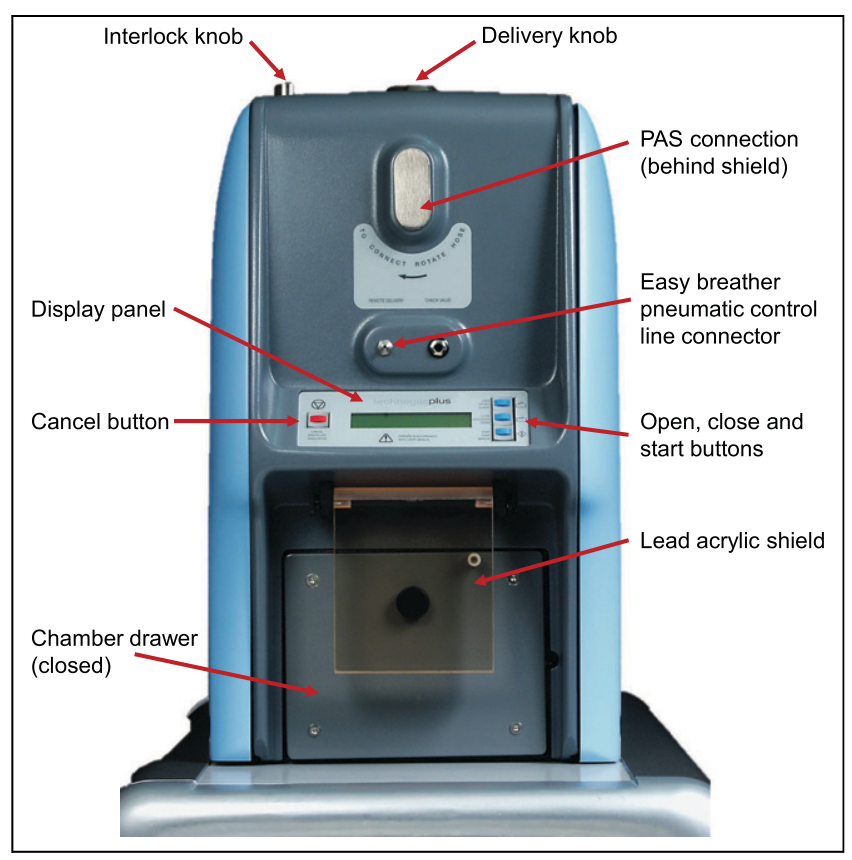

FIGURE 1. Annotated front view of Technegas generator. Chamber drawer is in closed position, and shield is closed down over administration set port. Not all models are fitted with easybreather option. PAS = patient-administration set. (Courtesy of Cyclomedica.)

in or adjacent to the radiopharmacy) and delivered under battery power to patients in an inpatient trolley/gurney bay, in an outpatient consultation room, on the $\gamma$-camera, or in a remote ward. The burn must be performed while the generator is connected to the main power supply, after which there is a 7- to 10-min window to complete patient ventilation; thus, remote delivery requires careful planning.

The second step is the simmer phase, in which the ${ }^{99 \mathrm{~m}} \mathrm{Tc}-$ sodium pertechnetate is evaporated in the crucible well, usually $10-30 \mathrm{~min}$ before the patient arrives. Before loading the new crucible, one must ensure that the previous crucible has fractured and dropped into the collection tray, which should be emptied before a new crucible is loaded, usually

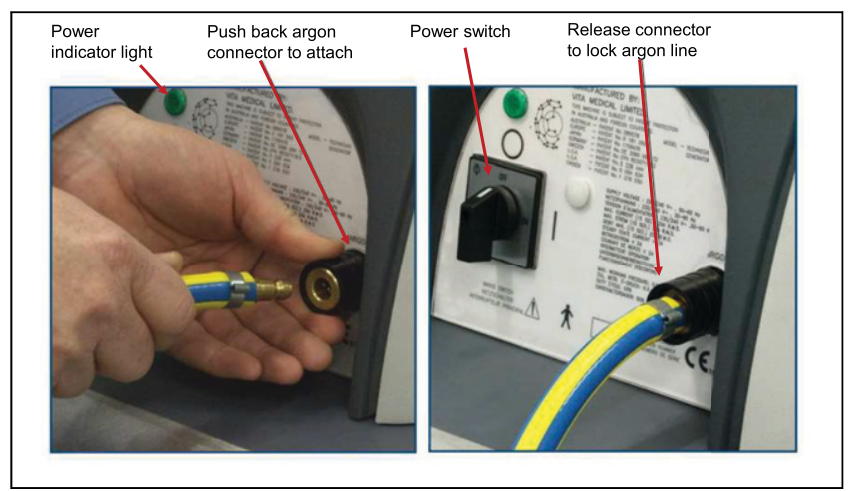

FIGURE 2. Annotated rear view of Technegas generator demonstrating connection of argon to system. (Courtesy of Cyclomedica.) 


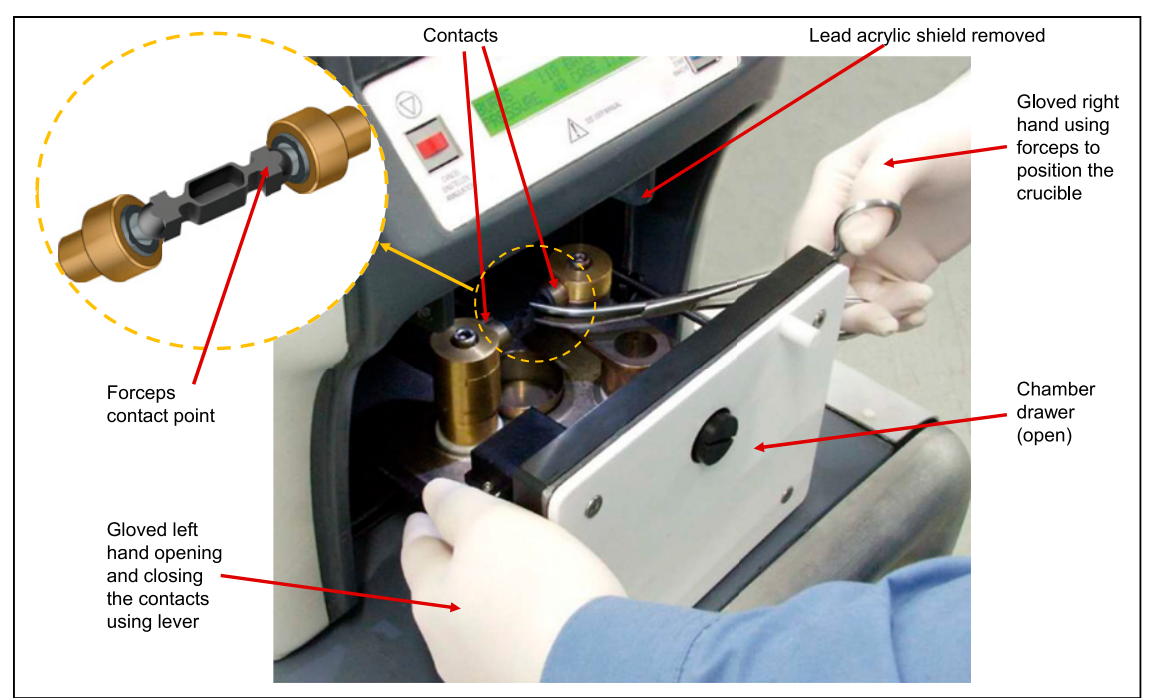

FIGURE 3. Use of gloves and good dexterity are required to place crucible between contacts, making good connection with fracturing crucible. For photography purposes, lead acrylic shield has been removed. (Courtesy of Cyclomedica.)
The third step is preparing the patient with a practice run. This step is no doubt among the most important in optimizing Technegas performance. The key to successful Technegas delivery is patient compliance, which is achieved through good patient education and practice, and should not be rushed. A detailed explanation of the ventilation process in language that the patient understands, with ample opportunity for clarification and questions, is essential. The process will be foreign to many patients, although those who are familiar with snorkeling may be more comfortable with it. The difficulty is compounded by patients' different levels of respiratory distress, which can produce a large variation in patient performance and thus affect ventilation image quality. The pracbefore arrival of the first lung scan patient of the day, to ensure that there is minimal radioactivity remaining. Visually inspecting the condition of the contacts and checking for any debris that might prevent the chamber from closing is also a useful step in preventative maintenance. The operator should avoid touching the parts of the crucible that will connect to the contacts because oil from the skin will reduce connection efficiency and Technegas yield. Loading the new crucible requires a degree of dexterity in manipulating it using forceps (Fig. 3). Because anything inside the chamber is potentially radioactive, gloves and standard radiation safety practices must be applied. The surfaces of the crucible well need to be wet with ethanol at the time the ${ }^{99 \mathrm{~m}} \mathrm{Tc}$ is added, or the ${ }^{99 \mathrm{~m}} \mathrm{Tc}$ volume may be displaced from the well during the simmer phase. The ethanol is generally applied before the crucible is loaded, but if the well is dry at the time the ${ }^{99 \mathrm{~m}} \mathrm{Tc}$ is added, the wetting can be repeated with the crucible in situ. The operator gently rotates the crucible back and forth between the contacts before adding the ${ }^{99 \mathrm{~m}} \mathrm{Tc}$ to ensure that a good connection is made to optimize Technegas yield. This rotation should not be a twisting motion, to avoid fracturing the fairly fragile crucible. The operator then adds $0.14-0.17 \mathrm{~mL}$ of ${ }^{99 \mathrm{~m}} \mathrm{Tc}-$ sodium pertechnetate containing $400-900 \mathrm{MBq}$. The meniscus should be flat or concave and not overflow the well (Fig. 4; top), as the ${ }^{99 \mathrm{~m}} \mathrm{Tc}$ would then simply be blown off the top of the crucible and a wet aerosol of free pertechnetate produced for inhalation, deteriorating image quality. If the total activity in $0.17 \mathrm{~mL}$ is lower than $400 \mathrm{MBq}$, multiple 6-min simmers can be performed to increase activity and avoid overloading the crucible volume. After ensuring that the argon pressure is optimized in the green zone of the regulator, the operator closes the drawer and commences the simmer. tice run should be done under the same conditions as the actual ventilation procedure in order to forestall any surprises. For example, if the patient practices while sitting but is lying down for the actual procedure, one can expect a difference in performance. Likewise, if the patient practices in a waiting area but undergoes the actual procedure on a

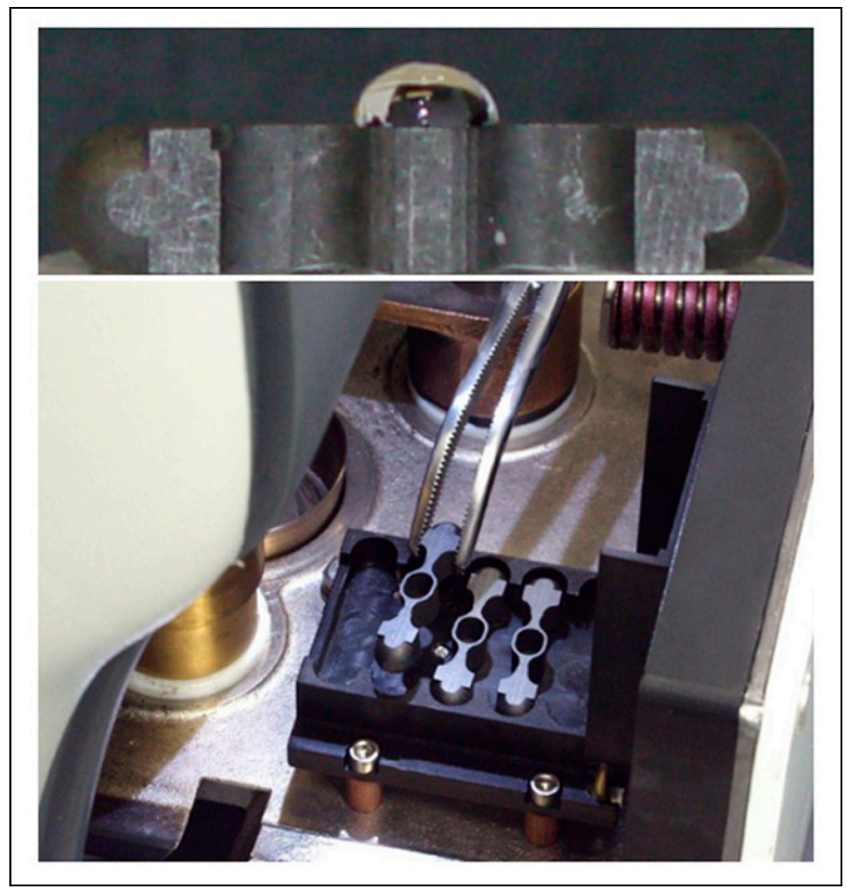

FIGURE 4. (Top) Excess ${ }^{99 m}$ Tc added to crucible well will be blown off during simmer and wasted. (Reprinted with permission of 18). (Bottom) Built-in simmer oven for crucibles in chamber to allow multiple simmer cycles for low-concentration ${ }^{99 m} \mathrm{Tc}$. (Courtesy of Cyclomedica.) 
TABLE 1

Technegas Tips

$1 \quad$ Performing ventilation upright produces distribution different from that when performed supine. ${ }^{99 \mathrm{~m}} \mathrm{Tc}$ macroaggregated albumin should be administered for perfusion in same position as that in which ventilation was performed.

2 Gloves and gown should be worn when performing the ventilation stage as this is biggest risk for contamination. This stage also requires face masks to be worn for coronavirus disease 2019 compliance.

3 For days on which eluate has high specific concentration, eluate should be diluted so that no more than $900 \mathrm{MBq}$ are contained in $0.15-\mathrm{mL}$ volume.

$4 \quad$ When used as per instruction, Technegas produces minimal contamination risk to staff and no risk of cross contamination between patients, a very important advantage with coronavirus disease 2019 (16).

5 Any unused crucibles in simmer oven at end of day can be cleansed with ethanol the next day and reloaded for simmer.

6 Since Technegas does not clear through kidneys, compared with ${ }^{99 \mathrm{~m}} \mathrm{Tc}$ -

diethylenetriaminepentaacetic acid aerosol studies, radiation dose to kidneys, bladder, and fetus is lower (17).

$\gamma$-camera, one might expect a difference in response, especially if the $\gamma$-camera makes the patient feel anxious. A common error is to have the patient practice without the nose peg, which creates an environment and level of compliance completely different from when the nose is blocked. The practice session improves timing, the ventilation process, patient compliance (especially with seals), and optimization of the appropriate mouthpiece for that patient (Fig. 5). Practicing the

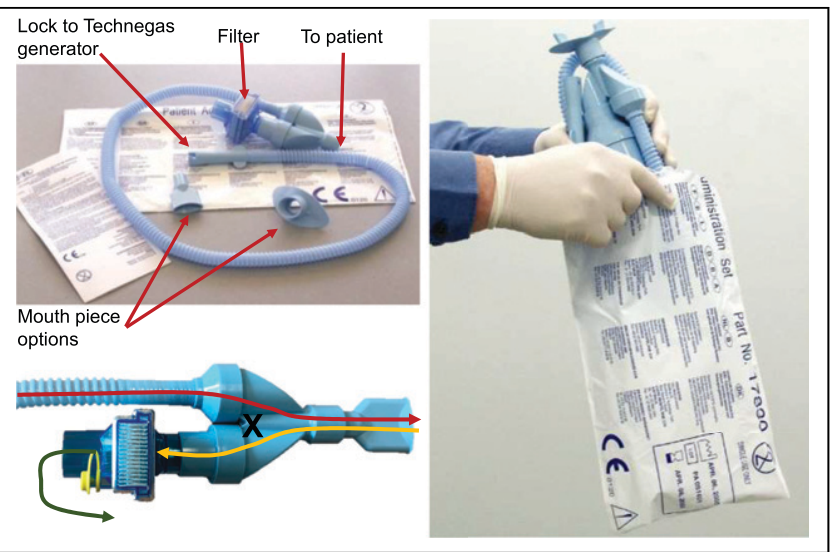

FIGURE 5. (Top left) Disposable administration set with multiple mouthpiece options to suit patient. (Bottom left) Administration set delivers Technegas to patient (red line) and returns patient breath through filter (yellow line) using 1-way valve to prevent communication between the 2 airflows (cross) and filtering both radioactive and biologic content of patient expiration to produce uncontaminated output into room air (green line). (Right) Entire administration set is disposed of in its original plastic bag. (Courtesy of Cyclomedica.) process is as much about preparing the patient as it is about making the administering staff aware of any compliance issues and the projected number of inspirations required.

The fourth step is to initiate the burn phase, which produces the combination of ${ }^{99 \mathrm{~m}} \mathrm{Tc}$ and carbon as nanoparticles under high temperatures in just $15 \mathrm{~s}$. This step should not be commenced until the patient is ready to be ventilated. As soon as the burn phase is completed, the Technegas should be delivered to the patient. The message "burn complete" is displayed, followed by an instruction to disconnect the generator from the main power supply. If the delivery is to take place at the production location, it is accomplished simply by switching the power button to off (Fig. 2). If the delivery is to be remote from the production location, the generator is disconnected from the main power supply and argon and transported to the patient.

The important fifth step is patient ventilation. The single most important inspiration is the first one, and this requires careful timing. The first release of Technegas (delivery knob) occurs under a small amount of pressure. The results are best when the release is timed during inspiration. If the release occurs during expiration or during the dead space at the end of inspiration or expiration, the high concentration Technegas in the first release is directed to the filter and wasted. The release should be timed to occur in the second quarter of the inspiration, and if practiced correctly, that inspiration will be a deep one. For that first inspiration, the patient will have been instructed to first breath out and then take a deep breath, hold for a second, and breath out. The operator should be providing these instructions during the delivery and should time the release with the first inspiration. The patient should return to normal-volume breaths after the initial inspiration, but care should be taken not to instruct the patient to "breath normally now," as patients have a tendency to do exactly that and break the seal or remove the apparatus from their mouth. The biggest risk of contamination comes when patients try to breath in or out around rather than through the mouthpiece. Perhaps "continue to breathe through the tube with normal-size breaths" is a better instruction. Once the ventilation portion of the test is completed, the patient should continue to breathe through the administration set to remove any remaining airborne Technegas residue for 4-5 breaths. If the delivery knob is not depressed, the system is diverted to breathing room air. After the ventilation portion of the test has deposited the desired amount of Technegas, the entire administration set is stored in its original plastic bag (Fig. 5 ) and disposed of as biologic waste after 10 half-lives.

The count rate from the lungs in a posterior orientation on the $\gamma$-camera should be about $1,000-1,500$ counts per second, which equates to between 20 and $50 \mathrm{MBq}$ delivered to the lungs. This allows a standard ${ }^{99 \mathrm{~m}} \mathrm{Tc}$-macroaggregated albumin administered dose to produce counts 4-7 times higher than the ventilation count. A perfusion count 4 times higher is the minimum to provide sufficient contrast to identify perfusion defects without being filled in by ventilation 
counts, whereas 7 times higher is optimal. Consequently, an over-efficient ventilation delivery could undermine diagnostic integrity. This situation might occur when a patient is highly compliant, with excellent inspiration, or when the ${ }^{99 \mathrm{~m}} \mathrm{Tc}$ is in high specific concentration. For example, fresh eluate from a ${ }^{99} \mathrm{Mo} /{ }^{99 m} \mathrm{Tc}$ generator early in the generator life cycle can have substantially more than $900 \mathrm{MBq}$ in $0.15 \mathrm{~mL}$ and, even in a suboptimal ventilation process, result in count rates in the lungs well in excess of 3,000 per second with 1 or 2 breaths. The long residence time of Technegas in the lungs means that radioactive decay is the only option to manage the count differential. A level of 3,000 counts per pixel would need to be delayed by a full half-life $(6 \mathrm{~h})$ before the perfusion is performed, for example. If the ventilation process is performed away from the $\gamma$-camera-with its instantaneous feedback on the amount deposited - then a radiation detector can be used to monitor the count rate in the lungs. In this case, the device should be calibrated against the $\gamma$-camera count rate so that specific readings on the radiation detector are known to equate to specific count rates on the $\gamma$-camera. Although it is common for the ventilation process to occur on the $\gamma$-camera itself, noncompliance during ventilation can contaminate the detector, increase the background count, and impact scanning for the remainder of the day.

The final step is the system purge, which takes a further 6 $\min$ and requires the Technegas generator to be reconnected to the argon and the main power supply. Residual Technegas in the chamber is purged through the system filter, after which the system is ready for the next simmer cycle. The purge - although occurring well within the acquisition window of the ventilation scan, let alone the entire V/Q study - is the rate-limiting step for a busy department. The patient-administration sets are single-use disposables and must be discarded between patients; however, on busy days with multiple ventilation scans scheduled, it is possible to use a single simmer phase and burn phase to ventilate more than 1 patient. Careful planning and timing are required, with each patient having an individual administration set. Because there is no biologic contamination from the patient into the Technegas system itself, changing administration sets between patients eliminates the risk of contamination between patients. The first patient ventilated should be the patient with the least compliance or greatest difficulties, to capitalize on the higher activity available for the first release. The residual can then be used for the second patient, who has a more optimal breathing status.

\section{LOW SPECIFIC CONCENTRATION OF ${ }^{99 M}$ TC}

There are many situations in which the ${ }^{99 \mathrm{~m}} \mathrm{Tc}$ eluate concentration may produce less than $400 \mathrm{MBq}$ in the $0.17-\mathrm{mL}$ crucible well volume. Some clinical departments do not have an on-site ${ }^{99} \mathrm{Mo} /{ }^{99 \mathrm{~m}} \mathrm{Tc}$ generator, and unit doses or bulk ${ }^{99 \mathrm{~m}} \mathrm{Tc}$ may have a low specific concentration, especially at the end of the day. Clinical sites operating with smaller generators may not produce a concentration sufficient to add $400 \mathrm{MBq}$ to the crucible in $0.17 \mathrm{~mL}$. For example, a generator eluted with $40 \mathrm{GBq}$ in $20 \mathrm{~mL}$ will, even at the time of elution, have only $300 \mathrm{MBq}$ in $0.15 \mathrm{~mL}$, which obviously decreases to $150 \mathrm{MBq}$ in the afternoon at $6 \mathrm{~h}$ after elution. Even departments with large generators can have low specific concentrations of ${ }^{99 \mathrm{~m}} \mathrm{Tc}$ toward the end of the weekly generator cycle. For example, a ${ }^{99} \mathrm{Mo} /{ }^{99 \mathrm{~m}} \mathrm{Tc}$ generator calibrated Monday with $120 \mathrm{GBq}$ might, by the end of the week, be expected to have a yield only on the order of $50 \mathrm{GBq}$ (accommodating elution efficiency) and, if eluted in $20 \mathrm{~mL}$, would produce less than $400 \mathrm{MBq}$ in $0.15 \mathrm{~mL}$ at elution time and less than $200 \mathrm{MBq}$ at $6 \mathrm{~h}$ after elution.

This problem has several possible solutions, with the best approach being dependent on the specific circumstances. The first option is to simply perform multiple simmers, adding ${ }^{99 \mathrm{~m}} \mathrm{Tc}$ to the crucible well between simmers. At the end of the simmer cycle, cancelling the process and opening the chamber drawer allows ${ }^{99 \mathrm{~m}} \mathrm{Tc}$ to be added because the volume has been reduced by evaporation, effectively doubling the total activity. This approach is perhaps the best for low-volume departments (in which a low specific concentration may impact only 1 or 2 patients per day) at the end of the day or toward the end of the generator week. It may also occasionally be necessary to do 3 or more simmer cycles (e.g., when the ${ }^{99} \mathrm{Mo} /{ }^{99 m} \mathrm{Tc}$ generator has a low yield at the end of the day or after hours). For those departments receiving bulk ${ }^{99 \mathrm{~m}} \mathrm{Tc}$ or unit doses from centralized radiopharmacies, a more efficient approach would be to purchase larger-volume crucibles. The standard crucible has a $0.15-\mathrm{mL}$ well that accommodates up to $0.17 \mathrm{~mL}$, whereas the larger crucibles have a volume of $0.3 \mathrm{~mL}$, accommodating enough ${ }^{99 \mathrm{~m}} \mathrm{Tc}$ to be equivalent to a double simmer cycle.

Some models of the Technegas generator have a built-in simmer oven that allows up to 5 crucibles to be mounted and loaded with ${ }^{99 \mathrm{~m}} \mathrm{Tc}$ and evaporated during each simmer cycle (Fig. 4, bottom). The crucible can have an additional $0.15 \mathrm{~mL}$ added to the first dose between simmer cycles or between full cycles of patient delivery. Because the crucibles are not in connection with the contacts, no arc is formed and no Technegas is produced during a burn cycle. An alternative approach that predates the built-in crucible ovens is an external crucible oven. These are simple hot plates, enclosed and shielded for radiation safety, in which up to 10 crucibles can be loaded with ${ }^{99 \mathrm{~m}} \mathrm{Tc}$ and evaporated multiple times. These are no longer commercially available but can be manufactured locally when a built-in oven is not available (the model for the U.S. market will not include the built-in crucible oven). In either case, the preloaded and preevaporated crucible is required to undergo a full simmer-and-burn cycle when placed between the contacts.

\section{TECHNEGAS VENTILATION IMAGING PROTOCOL}

After the patient has inhaled the Technegas to a rate of $1,000-1,500$ counts per second, imaging begins 


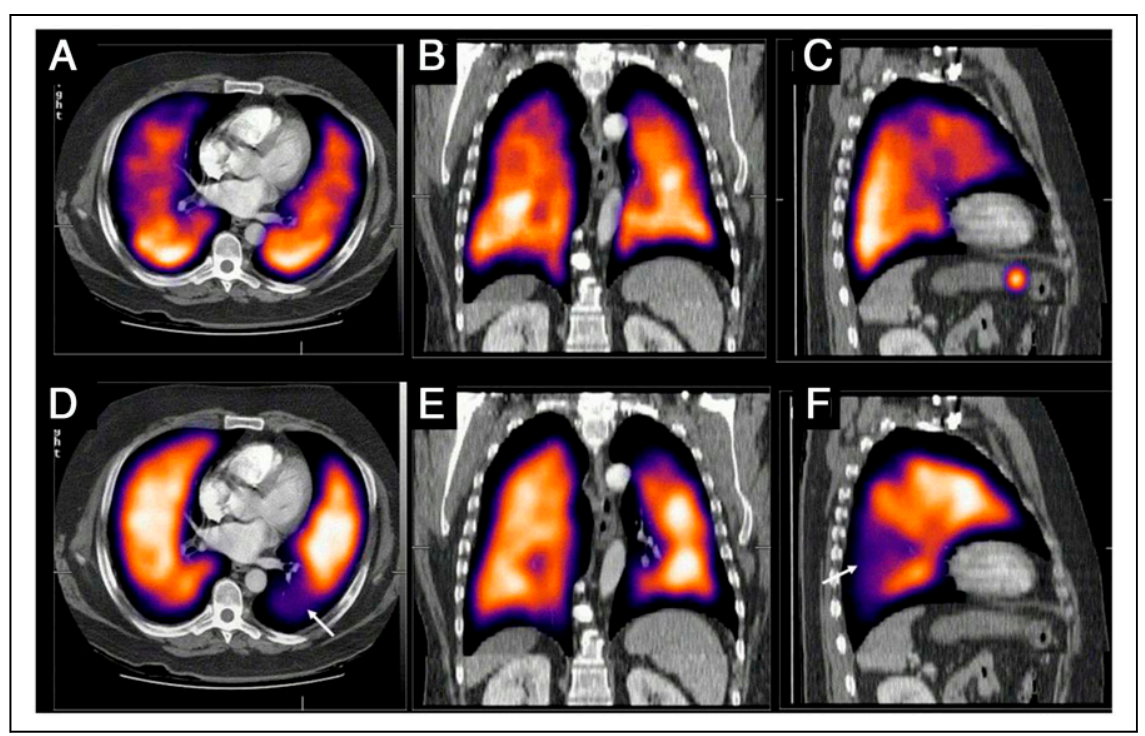

FIGURE 6. State-of-art contemporary SPECT/CT imaging of lung ventilation (top row) and perfusion (bottom row) in 3 orthogonal planes. SPECT/CT images demonstrate segmental perfusion defect (arrow in D and F) in left lower lobe. This appears mismatched when compared with corresponding ventilation images. Notch at apex of left lung seen in sagittal views ( $C$ and $F$ ) is matched on ventilation and perfusion and therefore is unlikely to be due to embolus.

immediately. Planar protocols (which are not recommended today, SPECT/CT being preferred) would involve 8 projections for $500,000-1,000,000$ counts, or 5 min per image (anterior/posterior; $45^{\circ}$ right anterior oblique $/ 45^{\circ}$ left posterior oblique; $45^{\circ}$ left anterior oblique $/ 45^{\circ}$ right posterior oblique; and left lateral/right lateral). The advised SPECT approaches include a minimum of 120 projections at $10-12$ $\mathrm{s}$ per projection with a $128 \times 128$ matrix, using a highresolution collimator despite the respiratory motion limits on spatial resolution. After iterative reconstruction, some sites produce planar-equivalent images from the dataset. One approach is to sum several projections on either side of the required view (anterior, for example, would be projection 1 summed with projections $2,3,119$, and 120) (4). A better approach is to reproject the SPECT data with an associated attenuation map (5). More commonly, however, the SPECT data provide the insights (Fig. 6) required and the planar extracted data are not required or produced. Although the principal application of the V/Q study is in the evaluation of known or suspect pulmonary embolism (12), Technegas ventilation studies are also useful in the evaluation of chronic obstructive pulmonary disease, chronic thromboembolic pulmonary hypertension, silicosis, cystic fibrosis, asthma, and emphysema, and in presurgical evaluation of regional variability in lung function, although a discussion of the clinical applications is beyond the scope of this article and can be found elsewhere (6).

\section{PERTECHNEGAS}

If the ultra-pure argon $(>99.99 \%)$ is adjusted with the addition of $2 \%-3 \%$ oxygen during the burn phase, the resulting carbon-bound ${ }^{99 \mathrm{~m}} \mathrm{Tc}$ produces the same gaslike distribution in the lungs but with rapid clearance from the lungs (7- to 10-min half-clearance time) $(1,13)$, referred to as pertechnegas. Because the standard Technegas has no effective clearance, it cannot be used for lung clearance studies. The oxygen-containing variant pertechnegas allows functional dynamic studies for lung clearance (13).

\section{GALLIGAS}

${ }^{68} \mathrm{Ga}$-chloride from a ${ }^{68} \mathrm{Ge} /{ }^{68} \mathrm{Ga}$ generator can be added to the Technegas generator in the same way as ${ }^{99 \mathrm{~m}} \mathrm{Tc}$ is used (no modifications) for ventilation PET imaging. This substance is commonly referred to as galligas. One important consideration for galligas is that the standard Technegas generator is designed to shield the $140-\mathrm{keV} \gamma$-emissions of ${ }^{99 \mathrm{~m}} \mathrm{Tc}$ and is therefore inadequate, from a radiation safety perspective, for shielding the $511-\mathrm{keV} \gamma$-emissions of ${ }^{68} \mathrm{Ga}(14) .{ }^{68} \mathrm{Ga}$-galligas can be used in combination with ${ }^{68} \mathrm{Ga}$-labeled microspheres to perform PET-based V/Q (15). This use exploits the advantages of PET/CT in terms of superior resolution; superior sensitivity; the capacity for respiratory gating, dynamic imaging, and quantification; decreased imaging time; and potentially decreased patient-absorbed radiation dose from the V/Q study (14), compared with SPECT imaging. Conversely, ${ }^{68} \mathrm{Ga}$ is more expensive and less readily available than ${ }^{99 \mathrm{~m}} \mathrm{Tc}$, even for those sites with a ${ }^{68} \mathrm{Ge} /{ }^{68} \mathrm{Ga}$ generator, and PET/CT is not as widely available as SPECT/CT and generally has a demanding oncology case load. However, galligas is an excellent tool for studying regional ventilation in respiratory physiology and research.

An alternative to galligas that emerged in conference abstracts in 2011 and 2012 was the potential for ${ }^{18} \mathrm{~F}$-fluorogas ventilation in combination with ${ }^{64} \mathrm{Cu}$-macroaggregated albumin. Details are scarce in the mainstream literature, and clinical use has not emerged. The obvious barrier to both ${ }^{68} \mathrm{Ga}$ and ${ }^{18} \mathrm{~F}$ PET imaging of the lungs is that the lungs are of low density, producing less attenuation of the $511-\mathrm{keV}$ photon emissions of ${ }^{68} \mathrm{Ga}$. In addition, the positron is of high energy (especially for ${ }^{68} \mathrm{Ga}$ ) and therefore travels some distance before annihilation, thus reducing spatial resolution (14).

\section{PRACTICAL PROTOCOL}

Operation of the Technegas system uses a stepwise procedure that is simple, safe, and effective (Fig. 7).

Step 1 is to check and load the generator: check that the main power supply and argon are connected, switch the power on, ensure that the argon regulator is in the green 


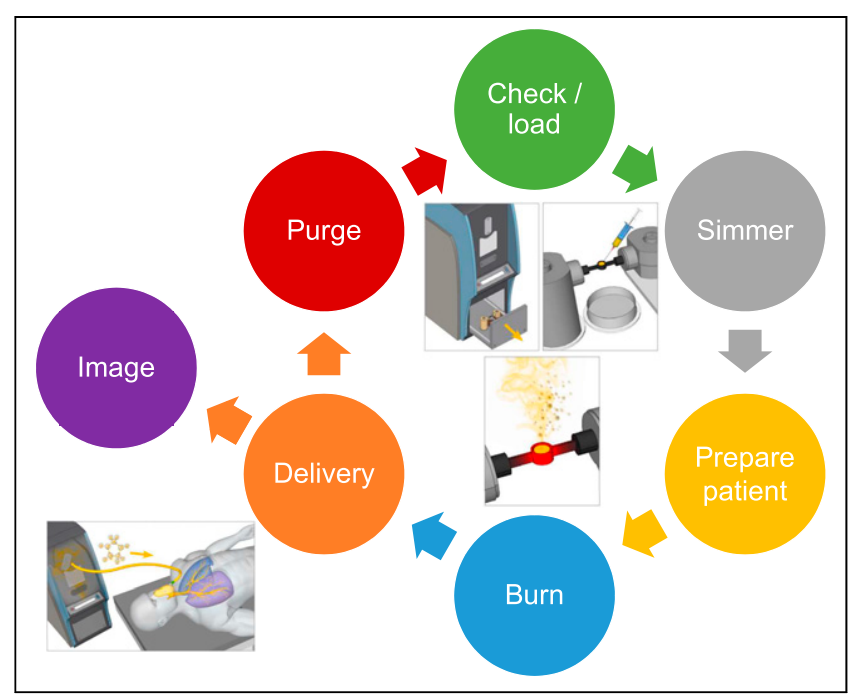

FIGURE 7. Technegas production cycle, beginning with checking and loading chamber. (Inset courtesy of Cyclomedica.)

zone, open the chamber drawer ("open" button), check for debris and whether the ash tray needs emptying, wet and drain the well of the crucible with ethanol, load the crucible between the contacts, add the ${ }^{99 \mathrm{~m}} \mathrm{Tc}$-pertechnetate to the crucible well, and close the drawer by simultaneously holding down the interlock knob and the "close" button until the chamber is fully closed.

Step 2 is to perform the simmer: press the start button to initiate the 6-min simmer cycle, and recheck the argon regulator to ensure that the pressure stays in the green zone as the argon purge is initiated.

Step 3 is to prepare the patient: identify the correct patient, explain the procedure, obtain informed consent, open a new administration set, have the patient practice breathing through the apparatus with specific attention to keeping an airtight seal, change the mouth piece if necessary, and identify and resolve any compliance issues.

Step 4 is to perform the burn: position and prepare the patient for Technegas delivery before initiating the burn, and press the "start" button to initiate the 15-s burn.

Step 5 is to deliver the Technegas: verify the burn, disconnect the main power supply and argon, transport the entire Technegas generator to the patient and connect the administration set to the system, ensure that the patient has the appropriate seal and press the "start" button to commence Technegas delivery, release the Technegas for patient ventilation by depressing the delivery knob during patient inspiration, and monitor the activity delivered with a target of 1,000-1,500 counts per second on the $\gamma$-camera or equivalent using a radiation detector.

Step 6 is to purge the system: confirm successful patient ventilation, return the Technegas generator to the main power supply and the argon supply and connect both, adjust the argon regulator to the green zone, and turn on the power switch so that the system cycles through a 6-min purge. At the end of the purge, the system is ready for the next patient cycle.
Step 7 is to obtain the images: on successful patient ventilation, commence the ventilation phase of the lung scan immediately, and dispose of the administration set.

\section{CONCLUSION}

Technegas is a simple yet versatile system for producing high-quality ${ }^{99 \mathrm{~m}}$ Tc-based ventilation studies. The design affords safety to patients and staff, including consideration of radiation and biologic risks. Technegas is the gold standard for ventilation studies for performing SPECT-based V/Q studies in pulmonary embolism and several respiratory pathologies. When approved by the U.S. Food and Drug Administration, Technegas will extend advantages to workflow, safety, and study quality for departments that adopt the technology.

\section{DISCLOSURE}

No potential conflict of interest relevant to this article was reported.

\section{REFERENCES}

1. Burch WM, Sullivan PJ, McLaren C. Technegas: a new ventilation agent for lung scanning. Nucl Med Commun. 1986;7:865-871.

2. McLaren CJ. Ventilation and perfusion lung tomography [abstract]. Aust $N Z J$ Med. 1987;17(suppl 2):459.

3. Rojas-Burke J. High hopes for Technegas. J Nucl Med. 1991;32(11):24N-25N, $29 \mathrm{~N}-30 \mathrm{~N}$.

4. Reinartz P, Schirp U, Zimmy M, et al. Optimizing ventilation-perfusion lung scintigraphy: parting with planar imaging. Nuklearmedizin. 2001;40:38-43.

5. Bailey DL, Schembri GP, Cooper RA, Bailey EA, Roach PJ. Reprojection of reconstructed V/Q SPECT scans to provide high count planar images [abstract]. J Nucl Med. 2005;46(suppl):337P.

6. Roach PJ, Bailey DL, Schembri GP, Thomas P. Transition from planar to SPECT V/Q scintigraphy: rationale, practicalities and challenges. Semin Nucl Med. 2010; 40:397-407.

7. Leblanc M, Tessier M, Ollenberger G, O'Brien C. CANM guidelines for ventilation/ perfusion (V/P SPECT) in pulmonary embolism. Canadian Association of Nuclear Medicine website. https://canm-acmn.ca/resources/Documents/Guidelines_Resources/ MasterDocument_Final_Nov_21_incl-Exec-Sum_ver3_Dec.\%2012_.pdf Published November 2018. Accessed September 8, 2021.

8. Roach PJ, Schembri G, Bailey DL. V/Q scanning using SPECT and SPECT/CT. J Nucl Med. 2013;54:1588-1596.

9. Roach PJ, Bailey DL, Harris BE. Enhancing lung scintigraphy with single photon emission computed tomography. Semin Nucl Med. 2008;38:441-449.

10. Roach PJ, Bailey DL, Schembri GP. Reinventing ventilation/perfusion lung scanning with SPECT. Nucl Med Commun. 2008;29:1023-1025.

11. Weibe L, Burch W, Abrams D. Technegas: ${ }^{99 \mathrm{~m}} \mathrm{Tc}-\mathrm{metal}$ core graphite nanoparticles for pulmonary ventilation imaging. Curr Radiopharm. 2010;3:49-59.

12. Bajc M, Olsson B, Gottsäter A, Hindorf C, Jögi J. V/P SPECT as a diagnostic tool for pregnant women with suspected pulmonary embolism. Eur J Nucl Med Mol Imaging. 2015;42:1325-1330.

13. Mackey DWJ, Jackson P, Baker RJ, et al. Physical properties and use of pertechnegas as a ventilation agent. $J$ Nucl Med. 1997;38:163-167.

14. Bailey DL, Eslick EM, Schembri GP, Roach PJ. ${ }^{68}$ Ga PET ventilation and perfusion lung imaging: current status and future challenges. Semin Nucl Med. 2016;46: 428-435.

15. Hofman MS, Beauregard JM, Barber TW, Neels OC, Eu P, Hicks RJ. ${ }^{68}$ Ga PET/ CT ventilation-perfusion imaging for pulmonary embolism: a pilot study with comparison to conventional scintigraphy. J Nucl Med. 2011;52:1513-1519.

16. Currie G. 2020, Post-COVID19 "new normal" for nuclear medicine practice: an Australasian perspective. J Nucl Med Technol. 2020;48:234-240.

17. Schembri GP, Miller A, Smart RC. Radiation dosimetry and safety issues in the investigation of pulmonary embolism. Semin Nucl Med. 2010;40:442-454.

18. Currie G, Wheat J, Traviss ZB. The impact of exceeding recommended crucible volumes in the Technegas generator. Internet J Nucl Med. 2006;3. 